\title{
Rydberg molecules in external fields: a semiclassical analysis
}

\author{
A. Matzkin and T. S. Monteiro \\ Department of Physics and Astronomy, University College London, Gower Street, London WC1E 6BT, U.K.
}

(November 5, 2018)

\begin{abstract}
We undertake a semiclassical analysis of the spectral properties (modulations of photoabsorption spectra, energy level statistics) of a simple Rydberg molecule in static fields within the framework of Closed-Orbit/Periodic-Orbit theories. We conclude that in addition to the usual classically allowed orbits one must consider classically forbidden diffractive paths. Further, the molecule brings in a new type of 'inelastic' diffractive trajectory, different from the usual 'elastic' diffractive orbits encountered in previous studies of atomic and analogous systems such as billiards with point-scatterers. The relative importance of inelastic versus elastic diffraction is quantified by merging the usual Closed Orbit theory framework with molecular quantum defect theory.
\end{abstract}

32.60.+i, 05.45.-a, 03.65.Sq

Gutzwiller Periodic Orbit theory (POT) [1] represents a very successful technique for establishing a correspondence between quantum properties and classically chaotic dynamics. Recently there has been much interest in diffractive POT, e.g. [2], for systems with a dynamical structure smaller or comparable to one de Broglie wavelength. These studies were restricted to a structureless point-scatterer, such as a simple $s$-wave scatterer. It is an interesting question, from the point of view of quantum chaology, to consider the effect of a diffractive scatterer with internal structure, such as for example a two-level particle. In fact we show here that this model provides us with the key to analyze semiclassically, for the first time, the spectra of diatomic Rydberg molecules such as $\mathrm{H}_{2}$ in static fields. We find that features such as the interference between Rydberg series converging on different energy thresholds depend on a novel type of inelastic diffractive trajectory.

Highly excited hydrogen atoms in static magnetic fields represents a well-known paradigm of quantum chaology: modulations in the quantum density of states are well described by POT [3]. However, analysis of the photoabsorption spectra from a low-lying initial state, require Closed-orbit theory (COT) [4], a semiclassical description of a density of states weighted by a dipole matrix element. Originally devised for hydrogen, COT was later extended to singly excited non-hydrogenic ('Rydberg') atoms [4.5], accounting for the presence of the ionic core. It was found [5] that the core-scattering produces additional 'combination' orbits. Non-hydrogenic energy-level spectra, even in weak fields where the classical dynamics for hydrogen is near-integrable, were found to be significantly more complex, showing short-range level repulsion and a proliferation of spectral modulations, a behavior termed 'core-induced chaos'. Subsequently, the nearest neighbor spacings (NNS) distributions of non-hydrogenic atoms in the 'core-induced chaos' regime were shown in fact [7], to correspond to a distribution which was neither Wigner (chaotic), nor mixed phase-space, but close to a new generic intermediate class known as semi-Poisson [8]. These findings were investigated experimentally for helium atoms in external fields [9].

We consider below the form of COT appropriate for simple molecules in external fields. The extension of COT is obtained by merging the standard closedorbit arguments with molecular quantum defect theory (MQDT), the main theoretical approach employed in the studies of Rydberg molecules 10]. The success of MQDT is based on two ingredients: the separation of the coreelectron interaction into long-range effects (described by Coulomb functions) and short-range effects (described by phase-shifts known as quantum defects), and the use of different basis sets to account for core-electron couplings. Thus, when the electron is near the core, its dynamics is coupled to the rotation of the core, the wavefunction being described in the coupled frame $|\alpha\rangle \equiv\left|\Lambda_{\alpha} l_{\alpha} J_{\alpha}\right\rangle$, ( $\Lambda$ is the projection of the electronic angular momentum on the molecular axis, $l$ is the orbital momentum of the outer electron, quantized along this axis, and $J$ gives the total angular momentum). For each $\alpha$, the quantum defect is given by the number $\mu_{\alpha}$ and scattering matrices have a simple representation, e.g. the $T$ matrix elements are $T_{\alpha}=e^{2 i \pi \mu_{\alpha}}-1$. When the electron is far from the core, the molecular wavefunction is described in the uncoupled frame $|j\rangle \equiv\left|N_{j} l_{j} m_{j}\right\rangle$ ( $N$ is the angular momentum of the freely rotating core, $l$ is now quantized in the laboratory frame along the axis of the magnetic field with magnetic quantum number $m$ ). These two coupling schemes are related by a unitary frame transformation with elements $\langle j \mid \alpha\rangle$. The $T$ matrix elements between outer channels $j$ and $j^{\prime}$ are given in terms of $T_{\alpha}$ by $T_{j j^{\prime}}=\sum_{\alpha}\langle j \mid \alpha\rangle T_{\alpha}\left\langle\alpha \mid j^{\prime}\right\rangle$. The frame transformation in the field-free case was given by the late U. Fano in a classic paper 11] which successfully analyzed experimental photoabsorption spectra for $\mathrm{H}_{2}$ from the ground state $(J=0, l=0)$ to high Rydberg states $(J=1, l=1)$. In the presence of a magnetic field of strength $\gamma$, the frame transformation is modified since $J$ is not conserved, but $M$, its projection on the field axis, is 12].

The quantum MQDT treatment, undertaken in an inner region where the magnetic field is negligible, is matched to the semiclassical one in the following way (see Fig. 1). (1) The molecule, initially in its ground state, is excited by a laser, giving waves with $l=1, J=1$, and both $\Lambda=0$ (phase-shifted by the quantum defect $\mu_{\Sigma}$ ) and $\Lambda=1$ (with a phase-shift given by $\mu_{\Pi}$ ). (2) As the electron leaves the core region, its dynamics gets uncoupled from the core which is left in one of the 4 allowed 
configurations: $N=0 m=0$ and $N=2 m=0, \pm 1$. These excited waves are propagated by the full field-free quantum molecular Green's function, obtained from the Lippmann-Schwinger form of the MQDT wavefunction 113 .

(3) The electron then enters the region in which the magnetic field is not negligible, where the total wavefunction takes the form $|\psi\rangle=\sum_{j} \sum_{N_{j}}\left|N_{j} M-m_{j}\right\rangle e^{i m_{j} \phi} \psi_{\text {out }}^{m_{j} N_{j}}$. Here, $\psi_{\text {out }}^{m_{j} N_{j}}$ is similar to the outgoing wave of the electron in COT [4]. For each core configuration, the electron's wavefunction is propagated in the magnetic field region semiclassically along classical trajectories $k$. Two dynamical regimes coexist, since the energy partition gives

$$
E_{N=0}^{e l}=E_{N=2, m}^{e l}+6 B_{r}+m \gamma / 2
$$

where $E^{e l}$ is the outer electron's energy and $B_{r}$ the core rotational constant (the dependence on $m$ through the Zeeman term for the $N=2$ levels is very small). (4) Eventually some trajectories return to the core. The molecular wavefunction is then written as a standard MQDT expansion, and the expansion coefficients may be obtained by matching the incoming part of the wavefunction to the semiclassical returning wave. (5) The electron is then projected onto the coupled frame, and the returning waves interfere with the initially excited waves to produce modulations in the absorption spectra.

These modulations contain contributions from the primitive closed orbits $k$ that left and returned to the core in one of its 4 possible configurations $j$ and are obtained by summing terms of the form

$$
\left[\sum_{\alpha, \alpha^{\prime}} \mathcal{C}_{\alpha} \mathcal{C}_{\alpha^{\prime}}\langle\alpha \mid j\rangle\left\langle j \mid \alpha^{\prime}\right\rangle e^{i \pi\left(\mu_{\alpha}+\mu_{\alpha^{\prime}}\right)}\right] \mathcal{A}_{k}^{j} e^{i S_{k}^{j}}
$$

where $\mathcal{A}_{k}^{j}$ and $S_{k}^{j}$ are the classical amplitude and action for trajectory $k$ and energy $E_{j}\left(S_{k}\right.$ includes the associated semiclassical phase, containing the Maslov index); $\mathcal{C}_{\alpha}=1$ if $\Lambda=0$ and $\sqrt{2}$ if $\Lambda=1$ is a coefficient stemming from the use of the united atom approximation for the dipole transitions. (6) The Coulomb field backscatters the returning waves which retrace the orbit in reverse, giving rise to repetitions without changing the state of the core. (7) Core-scattering produces newly outgoing waves, which are propagated in the outer region along orbits $q$. These waves now leave the core in state $N_{j} m_{j}$ but contain contributions from orbits that had previously returned with the core being in state $N_{j^{\prime}} m_{j^{\prime}}$, with the mixing strengths depending both on the classical dynamics (via the expansion coefficients) and the quantum scattering process (via the $T$ matrix elements). These orbits return to the core for the second time where they are matched once again to a MQDT expansion. The contribution of these orbits to the oscillatory part of the photoabsorption spectrum is of the form

$$
\begin{aligned}
& {\left[\sum_{\alpha, \alpha^{\prime}} \mathcal{C}_{\alpha} \mathcal{C}_{\alpha^{\prime}}\langle\alpha \mid j\rangle\left\langle j \mid \alpha^{\prime}\right\rangle e^{i \pi\left(\mu_{\alpha}+\mu_{\alpha^{\prime}}\right)} T_{j j^{\prime}}\right]} \\
& \times \mathcal{A}_{j}^{q} \mathcal{A}_{j^{\prime}}^{k} e^{i\left(S_{q}^{j}+S_{k}^{j^{\prime}}\right)}
\end{aligned}
$$

which clearly singles out the combination of an orbit $k$ at an energy $E_{j^{\prime}}$ with an orbit $q$ having an energy $E_{j}$. Further iterations may be performed, but since each process of matching the incoming wave to the MQDT expansion brings in a factor $\hbar^{1 / 2}$, fast convergence is expected for small $\hbar$.

COT for atoms has relied on exact or quasi-exact scaling properties since the classical dynamics of the excited electron depends only on a scaled energy $\epsilon=E \gamma^{-2 / 3}$. The field represents an effective value of Planck's constant $\hbar=\gamma^{1 / 3}$. Hydrogen in a magnetic field is nearintegrable for $\epsilon<-0.5$; as the field is increased it makes a gradual transition to full chaos at $\epsilon \simeq-0.1$. Although here the dynamics associated with the core in states $N=0$ and 2 cannot be scaled simultaneously, scaling techniques may still be applied theoretically to unravel the classical dynamics (e.g. 114). Eq. (11) is effectively scaled by giving a $\gamma^{2 / 3}$ dependence to the rotational constant thereby allowing calculations at fixed scaled energies $\epsilon_{N=0}$ and $\epsilon_{N=2}$. Full quantal calculations of the photoabsorption spectra were obtained by scaling the method described in [12,15. Note that $l$-mixing brings in (very weakly) the effects of $N=4,6 \ldots$ states. However their contribution is very small and although we include these in our quantal calculations, the physics remains in effect dominated by the $N=0,2$ series of quantum states. The $\mathrm{H}_{2}$ molecule and its isotopes $\mathrm{D}_{2}$ and $\mathrm{T}_{2}$ have similar quantum defects. We present the results for $\mathrm{T}_{2}$ below since its $B_{r}$ yields the smallest values of $\hbar$ in both channels $(\hbar \sim 1 / 90$ and $\sim 1 / 40$ for $N=0$ and $N=2$ respectively).

The Fourier transforms of the spectra are shown in Fig. 2 for $M=0$ at a scaled energy $\epsilon_{N=0}=-0.3$, corresponding to $\epsilon_{N=2}=-2.45$, over a range $\gamma^{-1 / 3}=60-120$. Over this range, $B_{r}$ varies from about twice to half its physical value. Each peak is associated with a closed orbit, either a 'geometric' orbit $k$ (i.e. one of the well-known 16 closed orbits of the diamagnetic hydrogen atom problem) appearing at scaled action $\widetilde{S}_{k}$ or a 'diffractive' combination of at least two orbits $k$ and $q$ at actions $\widetilde{S}_{k}+\widetilde{S}_{q}$. When $\mu_{\Sigma}=\mu_{\Pi}$, the terms between brackets in Eqs. (2) and (3) vanish for $N_{j}=2$ and $m_{j}=0, \pm 1$ (for any $j^{\prime}$ ) hence only peaks associated with the $\left(\epsilon_{N=0}\right)$ closed orbits are expected. For zero quantum defects [Fig. 2(a)] there is no core scattering (the $T$ matrix vanishes) and only geometric orbits are visible. For $\mu_{\Sigma}=\mu_{\Pi}=0.5$ [Fig. 2(b)], elastic core scattering between $\epsilon_{N=0}$ orbits produces the additional peaks; these combination orbits were seen in non-hydrogenic atomic spectra [5]. (c) shows $\mu_{\Sigma}=0.5$, $\mu_{\Pi}=-0.5$, in which case Eqs. (2) and (3) predict, as a generic feature, the geometric and elastic-scattered peaks corresponding to $\epsilon_{N=0}$ to be about 10 times weaker than the ones associated with the $\epsilon_{N=2}$ closed orbits. Moreover, the term given in Eq. (3) vanishes if $N_{j}=0$ and $N_{j^{\prime}}=2, m_{j^{\prime}}=0, \pm 1$, totally suppressing inelastic scattering. Indeed, the regularly spaced peaks in Fig. 2(c), are associated with the closed orbits of the near integrable $\epsilon_{N=2}$ case (the orbits perpendicular and parallel to the field for $\epsilon_{N=2}=-2.45$ have quasi-degenerate ac- 
tions) and their repetitions. Thus Figs. 2(a)-2(c) display simple features characterizing systems in which at most only elastic core-scattering is visible in the photoabsorption spectrum.

In contrast, the Fourier spectra shown in Figs. 2(d)(e) contain far more peaks. These arise from inelastic scattering between the core and the outer electron and appear at a scaled action corresponding to the sum of an $\epsilon_{N=2}$ orbit (or its repetitions) and an $\epsilon_{N=0}$ orbit. The shortest of these combination orbits occurs at $\widetilde{S}=1.71$ and combines the $V_{1}^{1}$ 'balloon orbit' [5] at $\epsilon=-0.3$ [peak ' 1 ' in Fig. 2(a)] with the primitive orbit 'i' at $\epsilon=-2.45$ [the first peak in Fig. 2(c)]. Note that two or more peaks having the same action but arising from different combinations will interfere constructively or destructively depending on the relative phases which contain both quantum scattering and classical orbit terms. However generic, peak-independent features are governed solely by the quantum defects. For example, when $\mu_{\Sigma}=0.5, \mu_{\Pi}=0$, the term between brackets in Eq. (3) is almost negligible when $N_{j}=N_{j^{\prime}}=0$, thereby effectively suppressing elastic scattering. This explains why in Fig. 2(d) there are no peaks corresponding to elastic core-scattering of the $\epsilon_{N=0}$ orbits. Fixing $\mu_{\Sigma}=0.5$, the height of these elastic peaks decreases monotonically when $\mu_{\Pi}$ is decreased from 0.5 to 0 , as expected by our semiclassical formulas. For $\mu_{\Sigma}=0.22$, $\mu_{\Pi}=-0.06$ [Fig. 2(e)], the quantum amplitudes linking the different channels have comparable magnitudes, and both elastic as well as inelastic combinations are seen.

We stress that the classical dynamics underlying Figs. $2(\mathrm{a})-(\mathrm{e})$ is identical, and the very different plots confirm the importance of the type of core scattering in the recurrence spectra. For example the $\mathrm{CO}$ molecule has equal quantum defects and its recurrence spectra should be similar to Fig. 2(b) (elastic scattering only). Fig. 2 (e) on the other hand corresponds to $\mathrm{H}_{2}$ which has the well-known quantum defects $\mu_{\Sigma}=0.22, \mu_{\Pi}=-.06$ at equilibrium internuclear distance.

We have also calculated the NNS distributions for different values of the quantum defects at $\epsilon_{N=0}=-0.5$. The results are given on Fig. 3. The distribution for $\mu_{\Sigma}=\mu_{\Pi}=0$ [Fig. 3(a)] appears as a superposition of 2 Poisson distributions, the first one concentrated at short-spacings $(s \simeq 0.3)$, the second one extending its tail at longer range. Note that although the Rydberg series do not interact, the overall spacing distribution is not equivalent to a superposition of 2 independent spectra (in which case the resulting distribution is well-known [17]). Nevertheless, it was possible to obtain an acceptable fit to the phenomenological formula $P(s)=\frac{\rho_{1}}{\rho}\left(\frac{\lambda}{\lambda-\rho_{2}}\right) P_{1}(\lambda, s)+\frac{\rho_{2}}{\rho}\left(\frac{\lambda-\rho}{\lambda-\rho_{2}}\right) P_{2}\left(\rho_{2}, s\right)$, with $\sum_{i} \rho_{i}=\rho$ where $\rho$ is the total density. $P_{1}$ gives the dominant distribution at short-spacings and vanishes for $s \gtrsim 1$, whereas $P_{2}$ gives the distribution which extends at larger spacings, with mean spacing $\rho_{2}^{-1} . \lambda$ is treated as a fitting parameter with $\lambda \geq \rho$. Thus for small spacings, $P(s)$ describes the superposition of $P_{1}$ and $P_{2}$ with the limiting cases $P(s) \rightarrow P_{1}(\rho, s)$ when $\lambda \rightarrow \rho$, and $P(s) \simeq \delta\left(s-\lambda^{-1}\right) \rho_{1} / \rho+P_{2}\left(\rho_{2}, s\right) \rho_{2} / \rho$ when $\lambda \rightarrow \infty$.

$P_{1}(s)$ was always taken to be a Poisson distribution, with $\lambda \simeq 4$. This is the consequence of the weak field experienced by the $N=2$ levels, which remain clustered around their field-free position. However, $P_{2}(s)$ does depend on the scatterer properties, i.e. on the quantum defects. For $\mu_{\Sigma}=\mu_{\Pi}=0, P_{2}$ follows a Poisson statistics (the tail of the solid line given in Fig. 3(a)). For $\mu_{\Sigma}=\mu_{\Pi}=0.5$ (b) the tail is in excellent agreement with the Wigner surmise, as is also the case for $\mu_{\Sigma}=0.5$, $\mu_{\Pi}=-0.5$ (c). On the other hand, for $\mu_{\Sigma}=0.5, \mu_{\Pi}=0$ [Fig. 3(d)] and for $\mu_{\Sigma}=0.22, \mu_{\Pi}=-0.06$ (e), $P_{2}$ is best approximated by a semi-Poisson distribution. Globally, $P_{2}$ goes from Poisson to Wigner, through intermediate statistics, as the intensity of the scatterer is increased. This intensity is given strictly by a multidimensional matrix obtained from the frame transformation as explained above [roughly, the intensity goes from 0 to a maximum value as $\left(\left|\mu_{\Sigma}\right|,\left|\mu_{\Pi}\right|\right)$ goes from $(0,0)$ to $\left.(0.5,0.5)\right]$. With our simple fit, the specific effects of inelastic scattering are not quantified. As known, 2 Rydberg series strongly interact when the levels cross each other, and we may thus expect the inelastic effects to appear at rather short spacings. We do remark however a smaller value of $\lambda$ for the cases (d) and (e), where inelastic scattering is stronger, which indicates a larger mean level repulsion for the distribution modelled by $P_{1}$.

In conclusion, we have shown that the relative contribution of geometric and diffractive trajectories produced by elastic and/or inelastic scattering on the core are essential to understand the character of recurrence spectra of different simple molecules in external fields. The spectral properties for these molecular systems, containing an effective 2-level scatterer, are dominated by 2 spacing distributions.

[1] M. C. Gutzwiller, Chaos in classical and quantum mechanics (Springer, New-York, 1990).

[2] H. Primack, H. Schanz, U. Smilansky, and I. Ussishkin, Phys. Rev. Lett. 76, 1615 (1996); H. Bruus and N.D. Whelan, Nonlinearity 9, 1023 (1996).

[3] H. Friedrich and D. Wintgen, Phys. Rep. 183, 37 (1989).

[4] M. L. Du and J. B. Delos, Phys. Rev. A 38, 1913 (1988); J. Gao, J.B. Delos, and M. Baruch, Phys. Rev. A 46, 1449 (1992).

[5] P. A. Dando, T. S. Monteiro, D. Delande and K. T. Taylor, Phys. Rev. Lett. 74, 1099 (1995).

[6] M. Courtney, H. Jiao, N. Spellmeyer and D. Kleppner, Phys. Rev. Lett. 73, 1340 (1994).

[7] T. Jonckheere ,B. Gremaud and D. Delande, Phys. Rev. Lett. 81, 2442 (1998)

[8] E. B. Bogomolny, U. Gerland, C. Schmidt, Phys. Rev. E 59, R1315 (1999).

[9] K. Karremans, A. Kips, W. Vassen, W. Hogervorst, Phys. Rev. A 60, R2649 (1999). 
[10] Ch. Jungen (Ed.), Molecular Applications of Quantum Defect Theory (Institute of Physics, Bristol, 1996).

[11] U. Fano, Phys. Rev. A 2, 353 (1970).

[12] T. S. Monteiro and K. T. Taylor, J. Phys. B 23, 427 (1990).

[13] A. Matzkin, Phys. Rev. A 59, 2043 (1999).

[14] J. Main, C. Jung and H. S. Taylor, J. Chem. Phys 107, 6577 (1997).

[15] X. H. He, K. T. Taylor and T. S. Monteiro, J. Phys. B 28, 2621 (1996).

[16] A. Holle, J. Main, G. Wiebusch, H. Rottke and K. H. Welge, Phys. Rev. Lett. 61, 161 (1988).

[17] T. Guhr, A. Muller-Groeling and H. A.Weidenmuller, Phys. Rep. 299, 190 (1998).

FIG. 1. Simplified view of the dynamics of photoabsorption of $\mathrm{H}_{2}$ in a magnetic field. The numbered steps are detailed in the text, but in brief: (1) A ground-state diatomic molecule absorbs a photon. (2) Outgoing waves with $l=1$ leave the core region; the dynamics of the corresponding excited electron uncouple from the core leaving it in a superposition of rotationally excited $(N=2)$ or not excited $(N=0)$ states. (3) In the extended region outside the core, the outgoing electron dynamics is described using classical trajectories. (4) Some trajectories return to the core carrying returning waves. (5) The returning waves interfere with the outgoing waves producing modulations in the spectra. Returning waves are either (6) back-scattered by the Coulomb field ('geometric' scattering in the terminology of diffractive POT) producing higher repetitions (7), or are core-scattered ('diffractive' scattering) without exchange of energy with the core (elastically) or with exchange (inelastically). The latter produce spectral modulations due to combinations of trajectories with different scaled energies (e.g. $\epsilon_{N=0}$ and $\epsilon_{N=2}$ ) and different classical regimes. They are not seen in atomic spectra and are a new feature, seen only in the molecular spectra.

FIG. 2. Fourier transformed quantum spectra for a set of diatomic molecules characterized by different quantum defects $\mu_{\Sigma}, \mu_{\Pi}$, showing the relative importance of elastic and inelastic diffractive trajectories. (a) $\mu_{\Sigma}, \mu_{\Pi}=0,0$ : no core scattering; peaks correspond to $N=0$ series (molecular core not rotationally excited / outer electron highly $(n \sim 90)$ excited) hence the COs correspond to those of a hydrogen atom at $\epsilon=-0.3$. (b) Shows only elastic core-scattering, within the $N=0$ series. (c) Spectra dominated by COs of $N=2$ states (molecular core rotationally excited / low excitation $(n \sim 40)$ electron) and elastic core scattering combining these trajectories. (d) Shows trajectories of both $N=0$ and $N=2$ states. New core scattered peaks are strongly dominated by inelastic diffractive trajectories combining $N=0$ and $N=2$ trajectories, i.e. the outer electron exchanges energy with the core. (e) $\mu_{\Sigma}, \mu_{\Pi}$ correspond to $\mathrm{H}_{2}$; shows mixture of elastic and inelastic core-scattered orbits.

FIG. 3. Spacing distributions (NNS) for different values of the quantum defects [linear scale for (a), log scale for (b)-(e)]. The solid line represents the fit $P(s)$. 

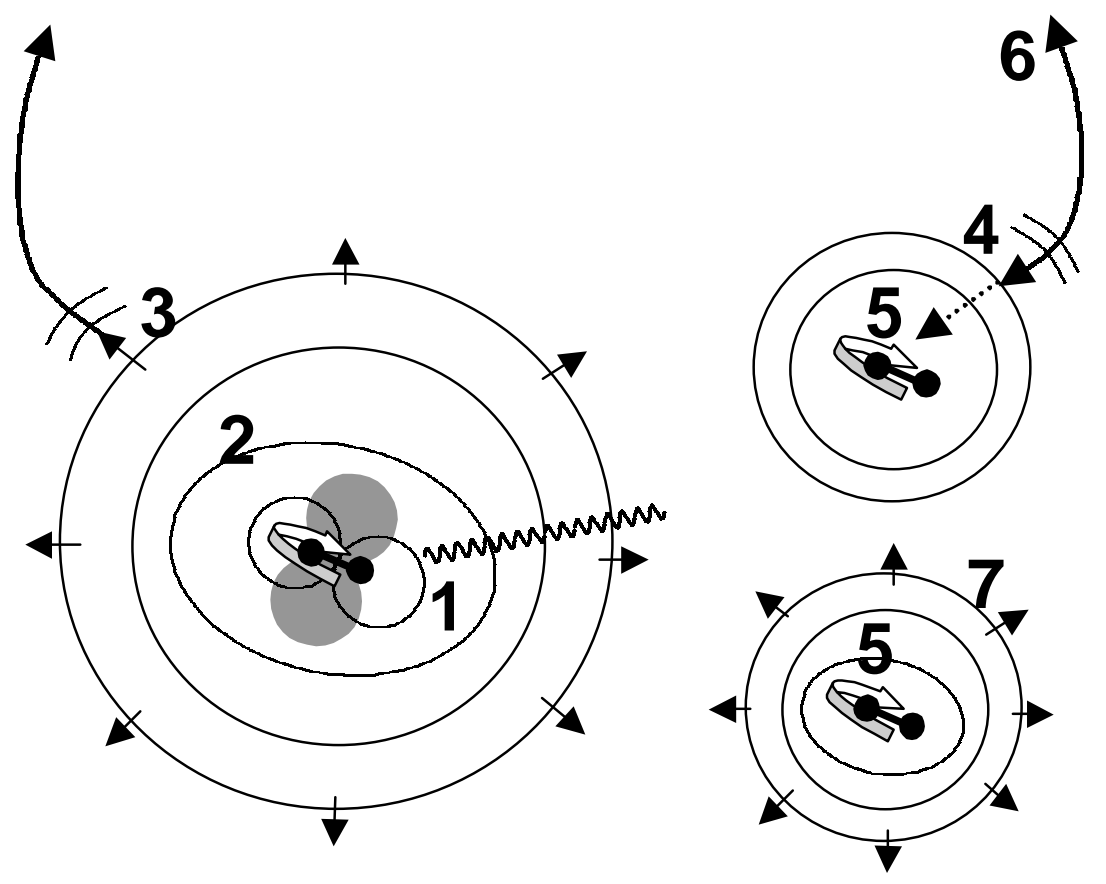




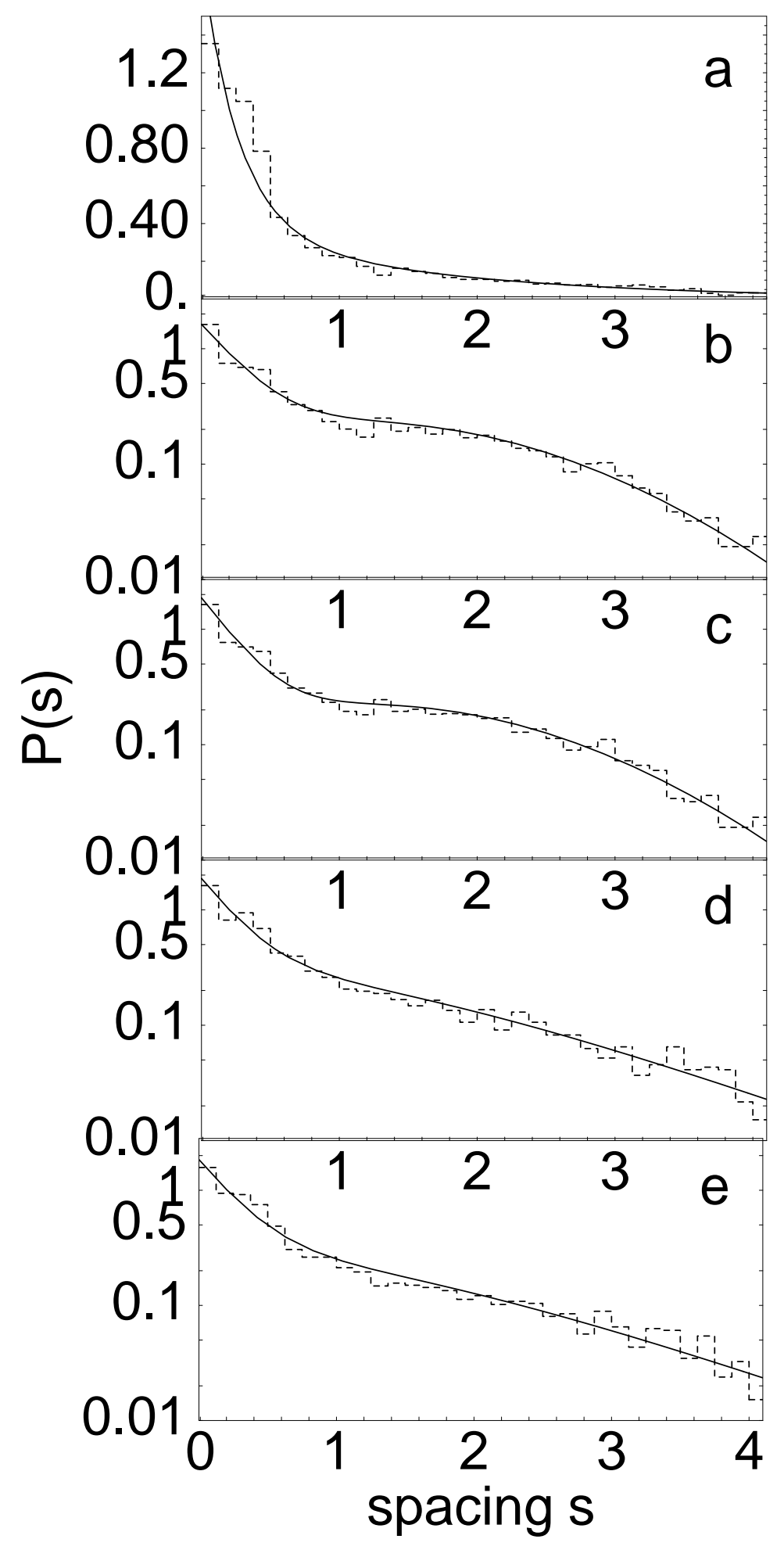

